\title{
Zur Präzision der Steuerprognose in Österreich
}

\author{
Markus Leibrecht \\ Institute of Public Sector Economics, \\ Vienna University of Economics and Business Administration, Austria
}

Zusammenfassung: Der Beitrag analysiert die Präzision der Aufkommensprognose wichtiger Bundesabgaben der Jahre 1976 bis 2002 in Österreich. Dadurch wird eine im Schrifttum bestehende Forschungs- und Informationslücke verringert. Eine Prognose wird dazu als präzise verstanden, wenn sie sowohl unverzerrt als auch im Mittel genau ist. Die Prognose des Steueraufkommens auf Bundesebene ist in Österreich gemessen am Bruttogesamtabgabenaufkommen präzise. Dennoch sind aufgrund der unpräzisen Prognosen wichtiger Einzelsteuern Verbesserungen möglich. Als mögliche Ursachen für die Verschätzungen werden die Organisation der Prognose, die verwendeten Prognosemethoden, der Vorsteuerbetrug, Ausgliederungstendenzen aus dem Staatshaushalt und neue kommunale Finanzierungsformen isoliert. Eine Erhöhung der Präzision sollte durch die Kombination mehrerer unabhängiger Prognosen zu einer Gesamtprognose, durch eine stärkere Dokumentation der Prognose, durch die Verwendung univariater Zeitreihenmethoden für die Prognose des Aufkommens an veranlagter Einkommensteuer und an Körperschaftsteuer und durch die Reduktion (Umsatzsteuer) bzw. Erhöhung (Mineralölsteuer) der verwendeten Aufkommenselastizitäten erreicht werden.

\begin{abstract}
The paper deals with the accuracy and the unbiasedness of tax revenue forecasting in Austria. It shows that the forecast performance greatly differs between the types of tax forecast. Whereas the aggregate tax revenue and the revenue from wage tax are forecast accurately and without bias the forecast of the revenue from the value added tax, the tax on corporate income, the personal income tax (self-employed labor) and from the petroleum tax are biased and/or not accurate. The paper isolates several possible reasons for this forecast performance, as, for example, problems with the statistical methods used, the organization of the forecast process and the tax fraud in the field of the VAT.
\end{abstract}

Keywords: Tax Revenue Forecasting, Accuracy, Unbiasedness, Austria.

\section{Einleitung}

Der Beitrag analysiert basierend auf Leibrecht (2004) die Präzision der Aufkommensprognose wichtiger Bundesabgaben der Jahre 1976 bis 2002 in Österreich. Dadurch wird eine im Schrifttum bestehende Forschungs- und Informationslücke verringert.

Im folgenden Abschnitt wird der in dieser Arbeit verwendete Begriff der Präzision einer Steuerprognose definiert und es wird eine Systematik von Determinanten der Präzision präsentiert. Abschnitt drei erläutert die in der Auswertung verwendeten statistischen Kennzahlen und Tests (Evaluationsmethoden). Abschnitt vier fasst die Evaluationsergebnisse 
zusammen. In Abschnitt fünf werden einige Ursachen für die ermittelte Präzision aufgezeigt. Abschnitt sechs bietet eine Zusammenfassung.

\section{Definition und Determinanten der Präzision}

\subsection{Definition einer präzisen Prognose}

Die Prognose von 1976 bis 2002 ist a posteriori präzise, wenn sie unverzerrt (unbiased) und im Mittel genau ist. Die Prognose ist unverzerrt, wenn der Erwartungswert des Prognosefehlers Null ist, d.h. falls

$$
\mathrm{E}\left(F_{t}\right)=\mathrm{E}\left(B R A_{t}-B V A_{t}\right)=0
$$

wobei $B R A_{t}$ den Realisationswert und $B V A_{t}$ den Prognosewert bezeichnen. Die Prognose ist im Mittel genau, wenn der Mittlere Absolute Prozentfehler der Prognose

$$
M A P F=100 \frac{1}{T} \sum_{t=1}^{T}\left|\frac{B R A_{t}-B V A_{t}}{B R A_{t}}\right|
$$

eine Schranke $S$ nicht übersteigt. Hier steht $T$ für den Beobachtungszeitraum. Bei Festlegung von $S$ sind der Prognosehorizont, die Art der Steuer sowie ihre fiskalische Bedeutung zu beachten (Leibrecht, 2004).

\subsection{Determinanten der Präzision}

Die Präzision einer Steuerprognose hängt von einer Vielzahl von Einflussfaktoren ab. Abbildung 1 fasst die wichtigsten Determinanten zusammen. Diese Systematik wird in Abschnitt 5 verwendet, um einige Ursachen für die ermittelte Präzision der Steuerprognose zu isolieren. Zum Folgenden siehe Leibrecht (2004) für Details.

Die Faktoren Steuerrecht und Änderungen des Steuerrechts, die konjunkturellen Rahmenbedingungen sowie das Verhalten der Zensiten bestimmen über vielfältige und zumeist interdependente Kanäle die Aufkommensentwicklung. Da diese Faktoren nicht direkt unter der Kontrolle der Steuerschätzung stehen (Ist-Determinanten), ist ihr gemeinsamer Einfluss anhand der Determinante Unsicherheit erfasst. Die verbleibenden Faktoren (Soll-Determinanten) stehen unter der direkten Kontrolle der Steuerschätzung. Institutionelle Faktoren beinhalten die Form der Verlustfunktion der Prognostiker (symmetrische oder asymmetrische Form) und die Möglichkeit des Vorliegens einer Art selbst erfüllender Prophezeiung. Organisatorische Faktoren umfassen die Anzahl der unabhängig voneinander prognostizierenden Institute und die Dokumentation der Prognose. Im Falle einer Prognose durch ein im Einflussbereich politischer Instanzen stehendes Institut ist die Möglichkeit eines Bias aufgrund politischer Einflussnahme auf das Prognoseergebnis zu bedenken. Als Ablauf der Prognose ist die strukturierte Vorgehensweise zur Ermittlung eines Prognosewertes definiert. Ein geeigneter Ablauf hat z.B. die Wirkungen von Steuerrechtsänderungen und der Konjunkturentwicklung zu berücksichtigen. Die Prognosemethoden sind eine weitere Determinante der Präzision, da einige Methoden gemäß der Literatur zum Prognosewettbewerb zu genaueren Prognosen führen als andere (Leibrecht, 2004). 


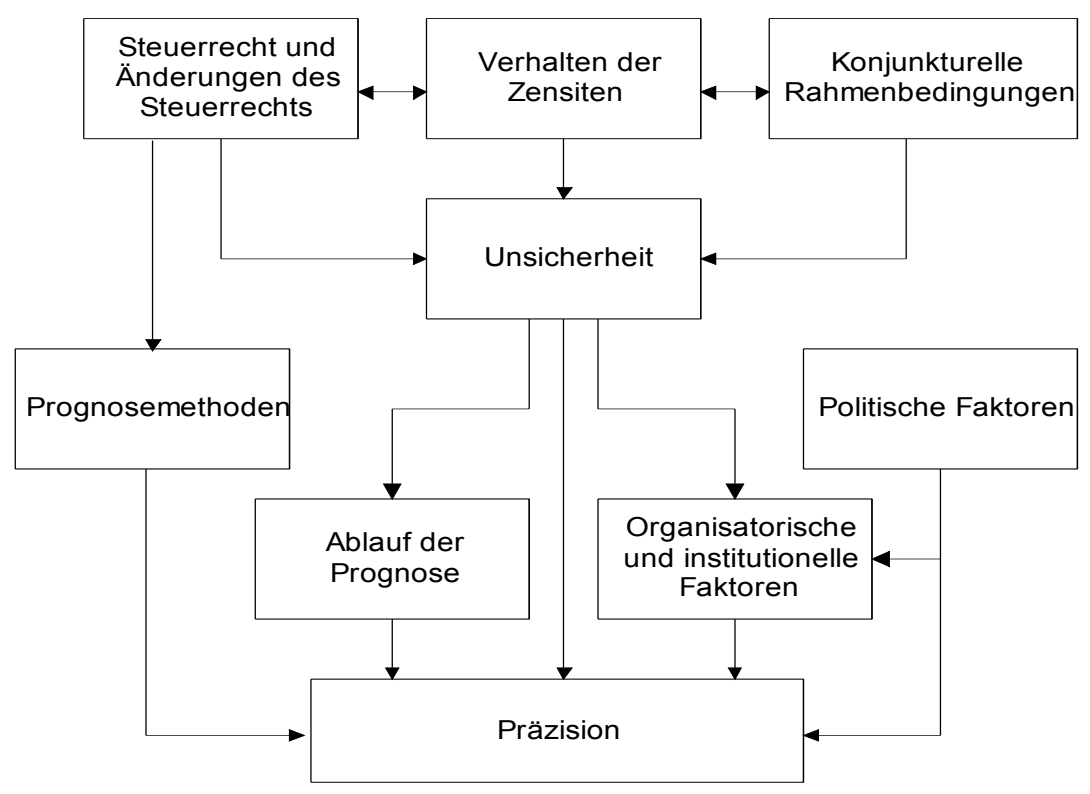

Abbildung 1: Systematik der Determinanten der Präzision (Quelle: Leibrecht, 2004).

\section{Evaluationsmethoden}

Neben $F_{t}$ und $M A P F$ liefert eine Reihe weiterer Kennzahlen aufschlussreiche Informationen zur Präzision der Prognose. Zudem sind Mittelwerttests auszuführen, um das Vorliegen einer unverzerrten Prognose zu bestimmen.

\subsection{Deskriptive Kennzahlen}

Der Prozentfehler

$$
P F_{t}=100\left(\frac{B R A_{t}-B V A_{t}}{B R A_{t}}\right)
$$

wird als Kennzahl verwendet, um die Prognosegenauigkeit für Jahre nach Steuerrechtsänderungen, mit Doppelbudgets und mit unerwarteten Rezessionen, zu evaluieren. Ist der Prozentfehler für diese Jahre systematisch größer als der $M A P F$, dann weist dies auf besondere Probleme der Steuerschätzung hin.

Der $U$-Koeffizient nach Theil

$$
U=\frac{\frac{100}{T-1} \sum_{t=2}^{T}\left|\frac{B R A_{t}-B V A_{t}}{B R A_{t}}\right|}{\frac{100}{T-1} \sum_{t=2}^{T}\left|\frac{B R A_{t}-B R A_{t-1}}{B R A_{t}}\right|}
$$

ist eine Kennzahl zum Vergleich der mittleren Prognosegenauigkeit der verwendeten Methode (Zähler) mit der mittleren Prognosegenauigkeit eines einfachen, symmetrischen 
„Random Walks“ (Nenner). Im Falle von $U>1$ ist die Prognose mittels Random Walk im Mittel genauer als jene mit der konkret verwendeten Methode (Mahmoud, 1987). Ein $U \geq 1$ ist demnach ein Indiz für die Wahl einer ungeeigneten Prognosemethode.

Der $M_{j}$-Koeffizient

$$
M_{j}=\frac{1}{\sigma_{j}^{B R A}} \sqrt{\frac{1}{T_{j}} \sum_{t=1}^{T_{j}}\left(B R A_{t j}-B V A_{t j}\right)^{2}}, \quad j=1,2, \ldots, K
$$

ist eine Kennzahl für die Veränderung der mittleren Prognosegenauigkeit im Zeitablauf, wobei hier die Wurzel des mittleren quadrierten Prognosefehlers (Root Mean Square Error, $R M S E$ ) als Indikator der mittleren Prognosegenauigkeit verwendet wird. Der Beobachtungszeitraum $T$ wird in mehrere vergleichbare Teilperioden $j$ geteilt. McNees (1992) folgend werden die einzelnen Perioden durch die Gewichtung des RMSE mit der Standardabweichung des Realisationswertes vergleichbarer. Sinkt der $M_{j}$-Koeffizient über die Teilperioden, dann liegt eine Verbesserung der Prognosegenauigkeit im Zeitablauf vor.

Der mittlerer Prognosefehler

$$
M F=\frac{1}{T} \sum_{t=1}^{T}\left(B R A_{t}-B V A_{t}\right)
$$

zeigt sowohl das Ausmaß als auch die Richtung einer möglichen Verzerrung der Prognose an. Ein statistischer Test auf die Unverzerrtheit der Prognose entspricht einem Test auf einen mittleren Prognosefehler von Null.

Die Theilsche Bias-Komponente

$$
B=\frac{\left(\frac{1}{T} \sum_{t=1}^{T}\left(B R A_{t}-B V A_{t}\right)\right)^{2}}{\frac{1}{T} \sum_{t=1}^{T}\left(B R A_{t}-B V A_{t}\right)^{2}}
$$

ist ein weiterer Indikator für das Vorliegen einer verzerrten Prognose. Eine unverzerrte Prognose weist eine Bias-Komponente kleiner 10 Prozent auf. Eine starke Verzerrung ist bei einem Wert von $B \geq 20$ Prozent anzunehmen (Pindyck and Rubinfeld, 1998).

Weist Steuer A einen höheren Variationskoeffizienten

$$
V=\frac{\sqrt{\frac{1}{T-1} \sum_{t=1}^{T}\left(B R A_{t}-\frac{1}{T} \sum_{t=1}^{T} B R A_{t}\right)^{2}}}{\frac{1}{T} \sum_{t=1}^{T} B R A_{t}}
$$

der Realisationswerte auf als Steuer B, dann ist A tendenziell schwieriger zu prognostizieren als B. 


\subsection{Testen der statistischen Signifikanz der Verzerrung}

Für eine statistisch abgesicherte Aussage zur Präzision ist der $M F$ auf einen statistischen Unterschied zu Null zu testen. Für die Wahl des geeigneten Mittelwerttests sind die Eigenschaften des stochastischen Prozesses von $F_{t}$ von zentraler Bedeutung. Im Speziellen sind folgende Eigenschaften von $F_{t}$ für die Wahl des geeigneten Mittelwerttests relevant: Verteilung von $F_{t}$ :

$$
F_{t} \stackrel{i d}{\sim} N\left(\mathrm{E}\left(F_{t}\right), \operatorname{var}\left(F_{t}\right)\right)
$$

mit $\mathrm{E}\left(F_{t}\right)<\infty$ und $\operatorname{var}\left(F_{t}\right)<\infty$.

Stationarität von $F_{t}$ : Für alle $s, t, k$ gelte

$$
\begin{aligned}
\mathrm{E}\left(F_{t}\right) & =\mathrm{E}\left(F_{t+s}\right), \\
\operatorname{var}\left(F_{t}\right) & =\operatorname{var}\left(F_{t+s}\right), \\
\operatorname{cov}\left(F_{t}\right) & =\operatorname{cov}\left(F_{t+s}\right) .
\end{aligned}
$$

Bedingung (1) verlangt, dass $F_{t}$ normalverteilt mit endlichem Erwartungswert und endlicher Varianz sowie frei von serieller Korrelation ist. (1) ist insofern als ,Idealbedingung“" anzusehen, da bei Gültigkeit ein einfacher T-Test ausgeführt werden kann. Bei Abweichungen von (1) sind alternative Mittelwerttests zu verwenden. Ist der Prognosefehler nicht seriell korreliert aber nicht normalverteilt, dann ist der T-Test nur asymptotisch gültig. In diesem Fall ist ein nicht-parametrischer Test mit höherer Macht in kleinen Stichproben vorzuziehen. Es kann ein Wilcoxon-Test (W) bei symmetrischer Verteilung von $F_{t}$ oder ein Vorzeichentest (VZ) bei nicht-symmetrischer Verteilung herangezogen werden (Conover, 1999). Ist der Prognosefehler seriell korreliert, dann ist das Testen unter Bonferroni-Grenzen (BF) möglich. Dazu wird die ursprüngliche Stichprobe in $z$ Teilstichproben geteilt, die frei von serieller Korrelation sind. Auf jede dieser Teilstichproben ist nun ein Mittelwerttest auszuführen. Wesentlich dabei ist, dass für jeden Test ein Signifikanzniveau von $\alpha / z$ angenommen wird, wobei $\alpha$ das Signifikanzniveau des Tests auf die ursprüngliche Stichprobe ist, und $z$ die Anzahl der neuen Stichproben angibt. Eine Prognose ist dann unverzerrt, wenn sie dies in jeder Teilstichprobe ist (Diebold and Lopez, 1996).

Die Bedingungen (2) - (4) sind hinreichend für das Vorliegen eines schwach stationären stochastischen Prozesses (Enders, 2004). Diese Bedingungen verlangen, dass der Erwartungswert und die Varianz von $F_{t}$ konstant und endlich sind und die Kovarianz zwischen den zwei Zufallsvariablen $F_{t}$ und $F_{t+k}$ nur vom Zeitraum $k$ zwischen den Zufallsvariablen abhängt. Erwartungswert, Varianz und Kovarianz müssen für schwache Stationarität demnach unabhängig von der Zeit sein. Wesentlich ist, dass ohne Stationarität des stochastischen Prozesses Mittelwerttests nicht sinnvoll sind.

Gebräuchliche Stationaritätstests sind der Dickey-Fuller-Test (DF) und der DickeyPantula-Test (DP) (siehe dazu Enders, 2004, und Harris and Sollis, 2003). Als Test auf serielle Korrelation ist der Breusch-Godfrey-Lagrange-Multiplikator-Test (BG) geeignet (Greene, 2003). Im Zuge der Ermittlung der Präzision einer Schätzung ist aufgrund des Problems ,sich überlappender Prognosen“ insbesondere die Möglichkeit des Vorliegens einer $M A(q)$-Form der seriellen Korrelation zu bedenken (Diebold and Lopez, 1996). Als Test auf Normalverteilung wird für geringe Stichprobenumfänge $(T \leq 50)$ der ShapiroWilks-Test (SW) empfohlen (Conover, 1980). 


\section{Evaluationsergebnisse}

\subsection{Datenbasis und Untersuchungszeitraum}

Evaluiert wird die Prognose des Bruttogesamtabgabenaufkommens des Bundes (BGA), der Umsatzsteuer (USt), der Lohnsteuer (LSt), der veranlagten Einkommensteuer (ESt), der Körperschaftsteuer (KöSt) und der Mineralölsteuer (MöSt). Die Daten sind dem Kapitel 52 der Bundesrechnungsabschlüsse (endgültige Realisationswerte) entnommen. Der Untersuchungszeitraum erstreckt sich für das BGA und die USt von 1976-2002, für die LSt von 1976-2001 (Wert von $F_{t}$ in 2002 wird als Ausreißer ausgeklammert; festgestellt anhand einer Box-Plot-Analyse der Verteilung von $F_{t}$ ), für die KöSt sowie die ESt von 1976-2002 ohne 2001 (Ausreißer) und für die MöSt von 1987-2001 (2002 ist als Ausreißer auszuklammern). Der verkürzte Beobachtungszeitraum für die MöSt ist notwendig, da das MöSt-Aufkommen bis 1986 in zwei Teilen prognostiziert wird. Einerseits als „Zweckgebundene“ MöSt, andererseits als „MöSt“. Der Beginn des Untersuchungszeitraums wird mit 1976 festgesetzt, da es Anfang der 1970ziger Jahre zu weitreichenden Änderungen in der Umsatz- und Einkommensbesteuerung kommt (Lehner, 1987, und Lehner, 1975).

\subsection{Definition von Prognosegenauigkeit}

Die Prognose ist der Literatur folgend (z.B. von der Lippe, 1998) im Mittel genau, wenn der $M A P F$ einen Wert um 2 Prozent oder darunter annimmt: $S \approx 2$ Prozent.

\subsection{Anmerkungen zu den verwendeten Kennzahlen und Tests}

$\mathbf{M}_{\mathbf{j}}$-Koeffizienten: Zur Berechnung der $M_{j}$-Koeffizienten wird der jeweilige Beobachtungszeitraum in 3 Teilperioden geteilt, wobei jede Periode zumindest einen gesamten Konjunkturzyklus und eine größere Steuerreform inkludiert. Es werden $M_{j}$-Koeffizienten für die Perioden 1976 bis 1984, 1985 bis 1993 und für die Periode beginnend mit 1994 berechnet. Für die MöSt liegt nur eine kurze Zeitreihe vor, die in zwei gleich lange Perioden, 1987 bis 1993 und 1994 bis 2001, geteilt wird.

Anmerkungen zu den ausgeführten Tests: Aufgrund des geringen Stichprobenumfangs wird stets auch ein nicht-parametrischer Mittelwerttest ausgeführt. Zudem wird das Signifikanzniveau mit Ausnahme des BG-Tests mit $\alpha=10$ Prozent angenommen. Für den BG-Test wird aufgrund seiner Eigenschaft, die Nullhypothese in kleinen Stichproben zu oft zu verwerfen (Verbeek, 2000), ein Signifikanzniveau von 1 Prozent angenommen.

Für die Ausführung des Dickey-Pantula-Tests wird eine maximale Einheitswurzel von $d=2$ angenommen. Alle Tests werden Said and Dickey (1984) folgend mit einer Lagstruktur von $T^{1 / 3}$ (ca. 3) begonnen. Anhand der Methode von Hall (Maddala and Kim, 1998) und des Informationskriteriums von Schwarz wird die Lagstruktur schrittweise bis zum optimalen Lag verringert. Die in die Schätzgleichung inkludierten deterministischen Komponenten werden Hamilton (1994) folgend gemäß Plot von $F_{t}$ gewählt und der Testsequenz in Enders (2004) folgend auf Signifikanz getestet. 


\subsection{Evaluationsergebnisse}

\subsubsection{Statistische Eigenschaften der stochastischen Prozesse}

Tabelle 1 ist zu entnehmen, dass die ausgeführten Stationaritätstests die Nullhypothese der Nicht-Stationarität zumindest auf dem 10-Prozent-Signifikanzniveau für jede Abgabe verwerfen. Mit Ausnahme der MöSt kann die Nullhypothese der Normalverteilung von $F_{t}$ nicht verworfen werden. Abgesehen von der KöSt ist $F_{t}$ frei von serieller Korrelation. Für die KöSt wird serielle Korrelation der 1. Ordnung angezeigt, sodass die Mittelwerttests unter Bonferroni-Grenzen mit 2 Teilstichproben (z1 und z2) und einem Signifikanzniveau von 5-Prozent durchgeführt werden.

Tabelle 1: p-Werte der Tests auf Stationarität, Normalverteilung und serieller Korrelation.

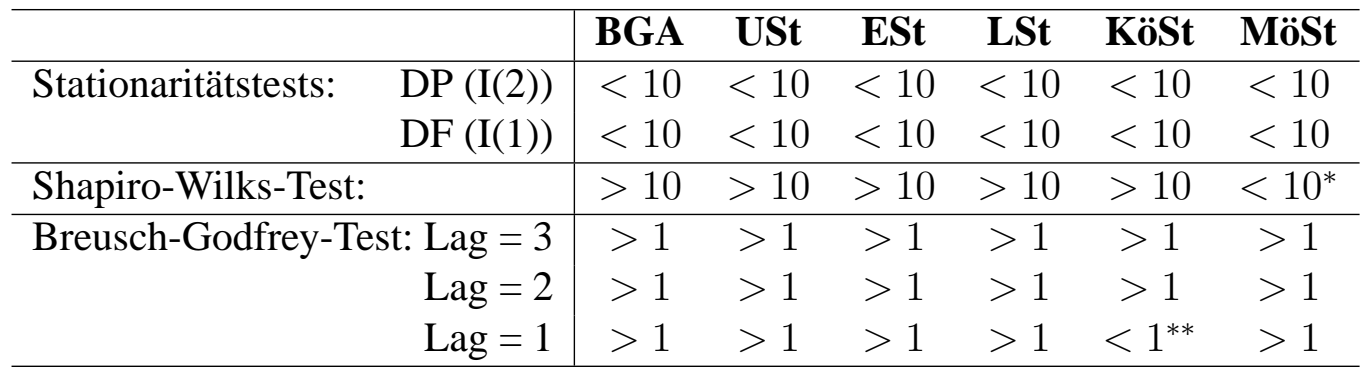

* signifikant bei $\alpha=10$ Prozent, ${ }^{* *}$ signifikant bei $\alpha=1$ Prozent.

\subsubsection{Präzision der Prognose der einzelnen Abgaben}

Die statistische Auswertung (Tabelle 2) zeigt, dass das Bruttogesamtabgabenaufkommen präzise prognostiziert wird. Der $M A P F$ liegt mit 2.2 Prozent nur knapp über der 2Prozentmarke und der $M F$ ist gemäß $B$-Wert sowie T- und $\mathrm{W}$-Test statistisch nicht von Null verschieden. Die Prognosegenauigkeit ist im Zeitablauf relativ konstant.

Das Lohnsteueraufkommen wird präzise prognostiziert. Der $M A P F$ liegt mit 2.25 Prozent nur knapp über der 2-Prozentmarke und die Prognose ist unverzerrt. Wie für das BGA ist die Prognosegenauigkeit im Zeitablauf relativ konstant.

Die Prognose des KöSt-Aufkommens ist unpräzise. Der $M A P F$ beträgt 10.14 Prozent, jedoch ist die Prognose gemäß $B$-Wert und den Mittelwerttests unverzerrt. Dies ist mit der hohen Volatilität der KöSt zu begründen $(V=81$ Prozent), die sich darin äußert, dass hohe Überschätzungen von hohen Unterschätzungen und umgekehrt abgelöst werden. Zudem hat sich die Prognosegenauigkeit im Zeitablauf verbessert, $\operatorname{der} M_{j^{-}}$ Koeffizient sinkt stetig.

Für die ESt zeigt die Auswertung eine unpräzise Prognose. Der $M A P F$ beträgt 6.30 Prozent und die Prognose ist statistisch signifikant verzerrt (Überschätzung). Der $U$ Koeffizient von 0.96 zeigt, dass ein einfacher Random Walk im Durchschnitt nicht zu ungenaueren Prognosen geführt hätte.

Der $M A P F$ der Prognose des USt-Aufkommens beträgt 2.61 Prozent, was, auch angesichts des relativ niedrigen Variationskoeffizienten der Ist-Werte, eine ungenaue Prognose impliziert. Die Prognose ist gemäß $B$-Wert bzw. T- und W-Test statistisch signifikant verzerrt (Überschätzung). Zudem ist eine Verschlechterung der Prognosegenauig- 
Tabelle 2: Deskriptive Kennzahlen und p-Werte des Mittelwerttests.

\begin{tabular}{|c|c|c|c|c|c|c|c|}
\hline \multicolumn{2}{|c|}{ Kennzahlen } & BGA & LSt & KöSt & $\mathbf{E S t}$ & USt & MöSt \\
\hline \multicolumn{2}{|c|}{$M A P F($ in $\%)$} & 2.20 & 2.25 & 10.14 & 6.30 & 2.61 & 3.26 \\
\hline \multicolumn{2}{|c|}{$P F($ in \%) 1984 (SR) } & 1.96 & & & & -0.37 & \\
\hline & 1989 (SR) & 1.17 & -4.50 & & & & \\
\hline \multicolumn{2}{|r|}{$1990(\mathrm{SR})$} & 2.26 & & -10.25 & 2.09 & & \\
\hline \multicolumn{2}{|r|}{$1992(\mathrm{SR})$} & & & & & 2.31 & \\
\hline & $1993(\mathrm{RZ})$ & -3.51 & -1.26 & -20.60 & -10.70 & -3.12 & 0.70 \\
\hline \multicolumn{2}{|r|}{1994 (SR) } & -1.24 & -2.40 & & & & \\
\hline \multicolumn{2}{|r|}{1995 (SR) } & -2.10 & & -6.70 & 2.90 & -5.00 & 8.08 \\
\hline \multirow{2}{*}{\multicolumn{2}{|c|}{$\begin{array}{l}1997 \text { (DP) } \\
1999 \text { (DP) }\end{array}$}} & -1.23 & -0.06 & -7.40 & 0.54 & -2.80 & 3.26 \\
\hline & & -1.68 & 2.46 & -7.43 & -5.40 & -2.60 & 5.62 \\
\hline \multicolumn{2}{|r|}{$2000(\mathrm{SR})$} & 1.24 & 2.55 & & & & \\
\hline \multicolumn{2}{|r|}{$2001(\mathrm{SR})$} & 3.05 & & & & & \\
\hline \multicolumn{2}{|c|}{$M F$ (Mrd. EUR) } & -0.175 & 0.030 & 0.010 & -0.076 & -0.199 & 0.042 \\
\hline \multicolumn{2}{|l|}{$B($ in $\%)$} & 5.00 & 1.70 & 0.00 & 26.30 & 32.40 & 25.00 \\
\hline \multicolumn{2}{|l|}{$V($ in $\%)$} & 42.77 & 47.40 & 81.37 & 27.44 & 38.04 & 28.70 \\
\hline \multicolumn{2}{|l|}{$U$} & 0.37 & 0.26 & 0.67 & 0.96 & 0.45 & 0.52 \\
\hline \multirow[t]{3}{*}{$M_{j}($ in $\%)$} & Periode 1 & 17.60 & 13.66 & 90.00 & 75.15 & 17.90 & 18.90 \\
\hline & Periode 2 & 14.00 & 16.50 & 38.00 & 35.70 & 15.80 & 37.60 \\
\hline & Periode 3 & 15.96 & 17.30 & 27.65 & 43.60 & 34.20 & \\
\hline \multicolumn{8}{|c|}{ Mittelwerttests } \\
\hline \multicolumn{2}{|l|}{ T-Test } & $>10$ & $>10$ & & $<10$ & $<10$ & \\
\hline \multicolumn{2}{|l|}{ W-Test } & $>10$ & $>10$ & & $<10$ & $<10$ & \\
\hline \multicolumn{2}{|c|}{ VZ-Test für MöSt } & & & & & & $<10$ \\
\hline \multicolumn{2}{|c|}{$B F$ für KöSt } & & & $\mathrm{z} 1 / \mathrm{z} 2$ & & & \\
\hline & W-Test & & & $>5$ & & & \\
\hline & VZ-Test & & & $>5$ & & & \\
\hline
\end{tabular}

keit seit 1994 festzustellen. Klammert man die Jahre mit besonders ungenauer Prognose (1994-2002) aus, dann ergibt sich ein etwas besseres Bild ( $M A P F=2.4$ Prozent, $B=24$ Prozent), das wesentliche Ergebnis (verzerrte und ungenaue Prognose) bleibt jedoch unverändert.

Auch das MöSt-Aufkommen wird unpräzise prognostiziert. Der $M A P F$ beträgt 3.26 Prozent. Dies ist auch deswegen beachtenswert, da die Variabilität im MöSt-Aufkommen relativ gering ist ( $V=28.7$ Prozent). Zudem wird das MöSt-Aufkommen gemäß $B$-Wert und VZ-Test statistisch signifikant verzerrt prognostiziert (Unterschätzung). Dies äußert sich auch darin, dass das Aufkommen lediglich in zwei Jahren überschätzt wird (1993 und 1995). Die Prognosegenauigkeit hat sich in Periode 1994 - 2001 deutlich verschlechtert, was auf die sehr hohe Verschätzung im Jahr 1995 ( $P F=8.08$ Prozent) zurückzuführen ist.

Die Auswertung zeigt weiters, dass umfassende Steuerrechtsänderungen (SR) und 
Doppelbudgets (DB) nicht systematisch - d.h. nicht für jede Steuer und auch nicht stets in $P F_{t}$ resultieren, welche den $M A P F$ übersteigen. Die unerwartete Rezession (RZ) von 1993 führt hingegen zu höheren Verschätzungen. Eine Ausnahme davon bildet lediglich die Mineralölsteuer. Hier ist für 1993 eine leichte Überschätzung des Aufkommens zu verzeichnen. Da das MöSt-Aufkommen jedoch statistisch signifikant unterschätzt wird, unterstreicht dieses Ergebnis die Bedeutung der konjunkturellen Rahmenbedingungen als Determinante der Präzision.

\section{Ausgewählte Ursachen für die ermittelte Präzision}

\subsection{Zur präzisen Prognose des BGA und der LSt}

Die präzise Prognose des BGA und des Lohnsteueraufkommens basiert wesentlich auf der Verwendung einer geeigneten Prognosemethode (zu den verwendeten Methoden siehe Leibrecht, 2004). Der Prognosewert des BGA ist die Summe der Prognosewerte der Aufkommen an den einzelnen Abgaben. Ein Vorteil dieser „Summierungsmethode“ ist, dass sich Über- und Unterschätzungen in den Einzelsteuern zum Teil kompensieren (Fehlerausgleich). So gleichen die leicht, wenn auch nicht systematisch, unterschätzte LSt und die systematisch unterschätzte MöSt die systematischen Überschätzungen der veranlagten ESt und der USt teilweise aus.

Das LSt-Aufkommen wird anhand eines Elastizitätenansatzes mit dem Durchschnittslohn (Elastizität von ca. 1.95) und den unselbständig Beschäftigten (Elastizität von 1) als Indikatorvariablen fortgeschrieben. Aufgrund des Vorliegens zeitnaher Steuerstatistiken, die es erlauben geeignete Elastizitätenwerte zu bestimmen und aufgrund der engen Verknüpfung der Indikatorvariablen mit der laut Steuerrecht relevanten Bemessungsrundlage der Lohnsteuer (siehe dazu z.B. Statistik Austria, 2001) ist der Elastizitätenansatz zur Prognose des LSt-Aufkommens geeignet. Zudem ermöglicht das Vorliegen zeitnaher Steuerstatistiken, die Effekte von Steuerrechtsänderungen relativ präzise zu quantifizieren.

\subsection{Zur unpräzisen Prognose der restliche Abgaben}

\subsubsection{Organisatorische Ursachen}

Anzahl der unabhängigen Prognosen: Zur Prognose des Status-Quo-Aufkommens werden zwar zwei unabhängig voneinander erstellte Prognosen (Prognose des Bundesministeriums für Finanzen und Prognose des Österreichischen Instituts für Wirtschaftsforschung) zu einer Gesamtprognose kombiniert, was den Empfehlungen der Literatur entspricht (Leibrecht, 2004). Es ist jedoch zu bedenken, dass sich die verwendeten quantitativen Prognosemethoden kaum unterscheiden und, dass das vorhandene Potenzial an unabhängig voneinander erstellten Prognosen nicht genützt wird. Bretschneider and Gorr (1987) folgend resultiert die Kombination unabhängiger Prognosen, insbesondere wenn sie mit verschiedenen Methoden durchgeführt werden, in einer Verbesserung der Präzision. Für eine institutionelle Neugestaltung der Steuerschätzung in Österreich könnte man sich am Arbeitskreis Steuerschätzung der Bundesrepublik Deutschland orientieren (Leibrecht, 2004). 
Offenlegung der Prognose: Die Steuerprognose ist in Österreich kaum dokumentiert. Die Offenlegung und Dokumentation der Prognose führt Bretschneider and Gorr (1987) folgend zu genaueren Prognosen. Das Fehlen von Informationen macht es unmöglich, die ausgewiesenen Prognosewerte intersubjektiv nachzuvollziehen. Damit verbunden ist auch, dass nicht an der Prognose beteiligte Personen die gemachten Annahmen nicht kritisch hinterfragen können. Auch sind deswegen einer gezielten Analyse der Ursachen von Verschätzungen enge Grenzen gesetzt. Eine stärkere Dokumentation der Prognose in Zukunft ist daher empfehlenswert.

\subsubsection{Prognosemethoden}

Die zur Schätzung verwendeten Methoden entsprechen großteils den aus der Literatur ableitbaren Empfehlungen zur Methodenwahl (siehe dazu Leibrecht, 2004). Nicht den Empfehlungen entspricht jedoch die Verwendung einer indirekten Methode, welche das Steueraufkommen als Funktion von erklärenden Variablen prognostiziert, zur Prognose des Aufkommens an ESt und an KöSt. Für beide Steuern liegen keine geeigneten erklärenden Variablen (Indikatorvariablen) vor (Leibrecht, 2004). Es sollte deswegen die Verwendung von direkten (univariaten) Zeitreihenmethoden für die Prognose angedacht werden.

Bezüglich USt und die MöSt ist festzuhalten, dass die verwendete Prognosemethode (Elastizitäten) zwar den Literaturempfehlungen entspricht. Jedoch sind die konkret verwendeten Werte der Elastizitäten zu gering (MöSt) bzw. zu hoch (USt). Da die MöSt bis auf 2 Jahre immer unterschätzt wird, sollten die verwendeten Elastizitäten in Bezug auf das reale BIP erhöht werden (derzeit Werte von 0.5 bis 1). Dies gilt vor allem für das Aufkommen aus Dieselverbrauch, das sich dynamisch entwickelt. Die USt wird hingegen systematisch überschätzt, sodass eine Reduktion der Elastizität in Bezug auf den privaten Inlandskonsum (gegenwärtig Wert von 1) zu einer präziseren Prognose führen sollte. Leibrecht (2004) inkludiert Schätzungen und Berechnungen der Elastizität mittels Kointegrationsanalyse und deskriptiv-statistischen Methoden. Je nach Modell und Methode ergeben sich Elastizitäten zwischen 0.72 und 0.88 .

\subsubsection{USt: Vorsteuerbetrug über Karussellgeschäfte}

Eine Ursache für die besonders deutlichen Verschätzungen des USt-Aufkommens seit 1994 liegt in dem durch den Binnenmarkt ausgelösten verstärkten Vorsteuerbetrug, dessen fiskalisches Ausmaß nur sehr ungenau prognostizierbar ist. Das Fehlen eines Grenzausgleichs öffnet Möglichkeiten zum Vorsteuerbetrug über ,,internationale Karussellgeschäfte“ (siehe dazu Peffekoven, 2001). Durch die Einführung des „Reverse Charge Systems“ (Mattes, 2002) sollte das Ausmaß des Vorsteuerbetrugs aber eingedämmt und die Prognose des USt-Aufkommens in Zukunft präziser werden.

\subsubsection{USt: Ausgliederungen und neue kommunale Finanzierungsformen}

Ausgliederungen aus dem Staatshaushalt ändern das Aufkommen an Umsatzsteuer, wenn die ausgegliederte Einheit vor Ausgliederung ein Hoheitsbetrieb ist, und nach Ausgliederung nachhaltig Einnahmen erzielt. In diesem Fall wird aus dem Hoheitsbetrieb ein 
marktwirtschaftliches Unternehmen mit Vorsteuerabzugsberechtigung. Dies dämpft das Aufkommen an Umsatzsteuer.

Auch neue Finanzierungsformen auf kommunaler Ebene, insbesondere Leasingfinanzierungen, haben Wirkungen auf die Bemessungsgrundlage der Umsatzsteuer. Eine Gemeinde kann z.B. bei Leasingfinanzierung die Vorteile des Vorsteuerabzugs geltend machen (Achatz and Leitner, 2001). Die fiskalischen Wirkungen von Ausgliederungen und neuen kommunalen Finanzierungsformen sind nur schwer prognostizierbar. Sie sind eine weitere Ursache für die besonders deutlichen Verschätzungen des USt-Aufkommens seit 1994, da sie seit diesem Zeitpunkt vermehrt eingesetzt werden.

\section{Zusammenfassung}

Die Prognose des Steueraufkommens auf Bundesebene ist in Österreich insgesamt - gemessen am Bruttogesamtabgabenaufkommen - präzise. Dennoch sind aufgrund der unpräzisen Prognosen wichtiger Einzelsteuern - Umsatzsteuer, Körperschaftsteuer, veranlagte Einkommensteuer und Mineralölsteuer - Verbesserungen möglich. Als mögliche Ursachen für die Verschätzungen werden die Organisation der Prognose, die verwendeten Prognosemethoden, der Vorsteuerbetrug, Ausgliederungstendenzen aus dem Staatshaushalt und neue kommunale Finanzierungsformen isoliert. Eine Erhöhung der Präzision sollte durch die Kombination mehrerer unabhängiger Prognosen zu einer Gesamtprognose, durch eine stärkere Dokumentation der Prognose, durch die Verwendung univariater Zeitreihenmethoden für die Prognose des ESt- und des KöSt-Aufkommens und durch die Reduktion (USt) bzw. Erhöhung (MöSt) der verwendeten Elastizitäten erreicht werden.

$\mathrm{Zu}$ beachten ist dabei stets, dass das Bruttogesamtabgabenaufkommen anhand einer „Summierungsmethode“ prognostiziert wird. Eine partielle, nur auf einzelne Abgaben gerichtete Verbesserung der Präzision der Prognose hat demnach unmittelbar auch Wirkungen auf die Präzision der Prognose des BGA. Eine umfassende Ursachenanalyse unter Einbezug aller wichtigen Abgaben ist daher notwendig.

\section{Literatur}

Achatz, M., and Leitner, R. (2001). Körperschaften öffentlichen Rechts und ihre Privatisierung im Steuerrecht. Wien: Verlag Österreich GmbH.

Bretschneider, S., and Gorr, W. (1987). State and Local Government Revenue Forecasting. In S. Makridakis and S. Wheelwright (Eds.), The Handbook of Forecasting: A Manager's Guide (p. 118-134). New York: Wiley \& Sons.

Conover, W. (1980). Practical Nonparametric Statistics (2nd ed.). Chichester: Wiley \& Sons.

Conover, W. (1999). Practical Nonparametric Statistics (3rd ed.). Chichester: Wiley \& Sons.

Diebold, F., and Lopez, J. (1996). Forecast Evaluation and Combination. In G. S. Maddala and C. Rao (Eds.), Handbook of Statistics (p. 241-268). Amsterdam: NorthHolland. 
Enders, W. (2004). Applied Econometric Time Series (2nd ed.). Chichester: Wiley \& Sons.

Greene, W. H. (2003). Econometric Analysis (5th ed.). London: Prentice Hall.

Hamilton, J. D. (1994). Time Series Analysis. Princeton: Princeton University Press.

Harris, R., and Sollis, R. (2003). Applied Time Series Modelling and Forecasting. Chichester: Wiley \& Sons.

Lehner, G. (1975). Gesamtwirtschaftliche Aspekte der Einkommensteuerreform 1975. Wirtschaftspolitische Blätter, 1, 24-40.

Lehner, G. (1987). Steuerpolitik in Österreich. Wien: Manz Verlag.

Leibrecht, M. (2004). Steuerschätzung in Österreich. Wiesbaden: Deutscher Universitätsverlag.

Maddala, G. S., and Kim, I. M. (1998). Unit Roots, Cointegration and Structural Change. Cambridge: Cambridge University Press.

Mahmoud, E. (1987). The evaluation of forecasts. In S. Makridakis and S. C. Wheelwright (Eds.), The Handbook of Forecasting: A Manager's Guide (p. 504-528). New York: Wiley \& Sons.

Mattes, N. (2002). Das Reverse Charge System (RCS), Teil 1. Finanz Journal, 11, 358-62.

McNees, S. K. (1992). How large are economic forecast errors? New England Economic Review, 25-33.

Peffekoven, R. (2001). Steuerhinterziehung bei der Umsatzsteuer - Wie kann dem Steuerbetrug begegnet werden? Die Steuerberatung, 9, Editorial.

Pindyck, R. S., and Rubinfeld, D. L. (1998). Econometric Models and Economic Forecasts (4th ed.). Boston: McGraw-Hill.

Said, E. S., and Dickey, D. A. (1984). Testing for unit roots in autoregressive-moving average models of unknown order. Biometrika, 71, 599-607.

Statistik Austria. (2001). Volkswirtschaftliche Gesamtrechnung 1976 bis 2000. Wien: Verlag Österreich GmbH.

Verbeek, M. (2000). A Guide to Modern Econometrics. Chichester: Wiley \& Sons.

von der Lippe, P. (1998). Steuerschätzung: Probleme, Methoden, Ergebnisse. Steuer und Studium, 5, 217-223.

Author's address:

Markus Leibrecht

Institute of Public Sector Economics

Vienna University of Economics and Business Administration

Augasse 2-6

A-1090 Vienna

Austria

E-mail: mleibrec@wu-wien.ac.at

http://www.wu-wien.ac.at/finanz 